\title{
Endothelialized flow models for blood transfusion research
}

\author{
Monica S.Y. Ng, ${ }^{1,2}$ Jacky Y. Suen, ${ }^{1}$ John-Paul Tung ${ }^{1,2}$ and John F. Fraser ${ }^{1}$
}

${ }^{1}$ Critical Care Research Group, Faculty of Medicine, University of Queensland, Brisbane and ${ }^{2}$ Research and Development, Australian Red Cross Blood Service, Brisbane, Australia

Haematologica 2019

Volume 104(3):428-434

\section{Correspondence:}

MONICA S.Y. NG

monica.ng91@gmail.com

Received: October 31, 2018.

Accepted: January 15, 2019.

Pre-published: February 14, 2019.

doi:10.3324/haematol.2018.205203

Check the online version for the most updated information on this article, online supplements, and information on authorship \& disclosures: www.haematologica.org/content/103/4/428

(C)2019 Ferrata Storti Foundation

Material published in Haematologica is covered by copyright. All rights are reserved to the Ferrata Storti Foundation. Use of published material is allowed under the following terms and conditions:

https://creativecommons.org/licenses/by-nc/4.0/legalcode. Copies of published material are allowed for personal or internal use. Sharing published material for non-commercial purposes is subject to the following conditions:

https://creativecommons.org/licenses/by-nc/4.0/legalcode, sect. 3. Reproducing and sharing published material for commercial purposes is not allowed without permission in writing from the publisher.

\section{Introduction}

Recent advances in cell culture and microfabrication technologies have enabled the development of perfusable endothelialized channels in vitro. To date, these techniques have primarily been applied to tissue engineering research. However, this set-up provides the unique opportunity to simulate blood product transfusion in a cost-effective, robust and reproducible manner - incorporating blood, endothelial and flow components in one unit. This Perspective describes the value of vascular models in transfusion research and discusses key decision points in the design process.

\section{What happens to blood after transfusion?}

On transfusion, blood products interact with blood cells and plasma components to alter platelet activation, leukocyte function and red blood cell (RBC) oxygen-carrying capacity. The pro-/anti-inflammatory balance hinges on whether neutrophil or macrophage responses dominate after blood product transfusion. ${ }^{1-3}$ These responses are dependent on the activation state of recipient neutrophils and macrophages which, in turn, is influenced by cytokines in the local microenvironment. RBCs and platelets modify immune system function by activating complement, releasing cytokines and participating in receptor-ligand interactions. ${ }^{4}$

Blood products and recipient blood are encased by endothelium in blood vessels, one of the largest organs in the body with a surface area of $350-1000 \mathrm{~m}^{2}{ }^{5}$ The endothelium conveys blood to tissues, provides a surface that prevents improper clotting and cellular activation, acts as a selective barrier to macromolecule extravasation and regulates microvascular blood flow. ${ }^{6}$ Activated endothelium participates in inflammation by releasing chemotactic molecules (e.g., interleukin8 and monocyte chemoattractant protein-1), generating reactive oxygen species and expressing adhesion molecules (CD62, CD106, CD54, CD31) to attract leukocytes and facilitate leukocyte transmigration. ${ }^{7,8}$ Furthermore, activated endothelium also enhances thrombosis by elaborating procoagulant surface molecules (von Willebrand factor, tissue factor) and microparticles. ${ }^{8}$ Endothelial dysfunction has been implicated in transfusion-related acute lung injury, sepsis and multiple organ dysfunction. ${ }^{8,9}$

The mixture of blood product and recipient blood is constantly mixed and propelled by the cardiac cycle - maximizing cellular interactions and minimizing inappropriate endothelial adhesion..$^{10}$ After leaving the heart, blood flows through arteries to reach capillaries in the tissues and then veins before returning to the heart. The three types of blood vessels differ in structure, diameter, flow patterns and shear stress. ${ }^{10}$ In arteries and veins, RBCs and leukocytes flow in the center of the flow stream and platelets are distributed to the periphery of the stream. ${ }^{11}$ RBCs exhibit a parabolic velocity profile with shear-dependent rotation which continuously mixes the blood components. The configuration of cells in the flow stream can be modified by RBC plasticity, shear rate and fluid viscosity. ${ }^{12,13}$ In the microvasculature, cells travel in single file with uniform distribution of platelets, RBCs and leukocytes in the flow stream.

Additionally, blood flow exerts shear stress on endothelium thereby altering endothelial gene expression, apoptosis, migration, permeability and alignment. ${ }^{6,14}$ Endothelial cells cultured under flow conditions demonstrate enhanced barrier function in conjunction with minimal adhesion molecule activation. . $^{15,16}$ Physiological shear stress is protective against inappropriate endothelial cytokine release compared to low shear stress. ${ }^{9}$ Additionally, flow patterns (e.g. continuous versus pulsatile) influence endothelial adhesion protein expression, structure and 
Table 1. Available models for testing blood-endothelial interactions in vitro.

\begin{tabular}{|c|c|c|c|}
\hline $\begin{array}{l}\text { Model } \\
\text { Static conditions }\end{array}$ & Protocol & Outcome measures & Research output and clinical impact \\
\hline Blood unit sampling & $\begin{array}{l}\text { - Repeated sampling of blood product } \\
\text { over shelf life (37-41) }\end{array}$ & $\begin{array}{l}\text { - Markers of platelet activation } \\
\text { - apoptosis (37) } \\
\text { - Media change (38) } \\
\text { - Microparticle accumulation (38) } \\
\text { - Proteomics (42) } \\
\text { - Procoagulant characteristics (39) } \\
\text { - RBC anti-oxidant oxidation during } \\
\text { storage (40) } \\
\text { - Membrane deformability (41) }\end{array}$ & $\begin{array}{l}\text { - PC undergo deterioration of mitochondrial } \\
\text { integrity + apoptosis (37) during storage } \\
\text { - PRBC storage leads to enhanced RMP } \\
\text { formation, osmotic fragility, hemolysis, } \\
\text { decreased deformability (38), } \\
\text { prolonged activated clotting time (39), } \\
\text { increased RBC anti-oxidant oxidation (40) }\end{array}$ \\
\hline
\end{tabular}

Specific component co-incubation
- Blood products or supernatant of blood product mixed with purified cell populations from healthy volunteers - Neutrophils $(1,17-21)$

- Macrophages (3)

- Platelets (22)

- HUVECs (23)
- Neutrophil apoptosis (17)

- Neutrophil ROS formation $(17,19,20)$

- Neutrophil activation markers $(1,18,21)$

- Neutrophil phagocytosis (1)

- Macrophage cytokine elaboration (3)

- Platelet adhesion + aggregation (22)

- RBC adhesion to endothelial cells (23)
- Supernatant from stored PRBCs delays neutrophil apoptosis and primes neutrophils (17)

- PMP binding to neutrophils increases CD11b expression + phagocytic activity (1)

- Non-polar lipids accumulated during ex vivo PRBC storage prime neutrophils (20)

- PRBCs stored for prolonged periods are less capable of supporting platelet adhesion + aggregation (22)
Whole blood incubation • Blood product components incubated with whole blood $(2,24)$

- Storage-induced RMP incubated with whole blood (43)
- Cytokine production after adding fresh or stored PRBC supernatant to whole blood $(2,24)$

- Thromboelastometry, microparticle characterization, cytokine concentration (43)
- Incubation of microparticles from PRBCs with whole blood induced host production of TNF, IL-6, IL-8 (2)

- RMPs from stored PRBCs trigger coagulation of whole blood through TF signaling (43)

\section{Laminar flow conditions}

Acellular planar $\quad$ - Cells from blood products perfused flow model over layer of subendothelial matrix or collagen (44)
- Influence of PC storage duration on adhesion capacity to subendothelial matrix + collagen (44)
- Platelet aggregation + adhesion capacity are improved in $\mathrm{BC}$ PC compared to PRP PC (44)
Cellular planar flow model
- Endothelial cells cultured on coverslips and subjected to specific shear stress

$-0.3 \mathrm{dyn} / \mathrm{cm}^{2}(27)$

$-5 \mathrm{dyn} / \mathrm{cm}^{2}(26)$

- $10 \mathrm{dyn} / \mathrm{cm}^{2}$ (25)

- Shear stress not reported (25)

- Different mixtures of blood perfused

through system

- 10\% whole blood suspension (25)

- $1.5 \%$ PRBC suspension $(26,27)$

- Use of activated endothelium

- E. coli 0127:B8 LPS (45)

- TNF- $\alpha(25,45)$
- Adhesion of RBCs to endothelium from PRBCs stored for different periods $(26,27,45)$

- Tissue factor expression + adhesion to endothelial cells (25)
- RBCs from stored PRBCs more adherent to HUVECs under laminar flow conditions compared to those from fresh

PRBCs $(26,27)$

- Exposure to TNF-stimulated HUVECs under arterial flow conditions led to increased tissue factor expression on monocytes compared to quiescent HUVECs (25)

- RBCs more adherent to $E$. coli-activated endothelium compared to quiescent endothelium (45)
Ex vivo artery model $\quad$ Human umbilical artery (up to $20 \mathrm{~cm}$ ) and connected to circuit (28)

- Whole blood diluted 1:10 circulated with shear pressure of $0.4 \mathrm{dyn} / \mathrm{cm}^{2}$

- Endothelium activated with TNF- $\alpha$
- Microparticle formation, endothelial ROS formation (28)
-Whole blood perfused through TNF-stimulated umbilical arteries demonstrated increased microparticle formation. These microparticles enhanced HUVEC ROS formation in vitro (28)
Synthetic arterial model • Biodegradable tubular scaffold matrices (length $4 \mathrm{~cm}$, inner diameter $6 \mathrm{~mm}$ ) seeded with HUVECs (29)
- Confocal microscopy, biochemical extracellular matrix analysis, endothelial nitric oxide formation (29)
- Monocytes circulated through the TNF-stimulated model adhered to the endothelium and transmigrated into the intima (29) 
continued from the previous page

\begin{tabular}{|c|c|c|c|}
\hline $\begin{array}{l}\text { Model } \\
\text { Static conditions }\end{array}$ & Protocol & Outcome measures & Research output and clinical impact \\
\hline $\begin{array}{l}\text { Acellular synthetic } \\
\text { microvascular model }\end{array}$ & $\begin{array}{l}\text { - Soft lithography with vessel diameters } \\
\text { of } 5-70 \mu \mathrm{m} \text { in PDMS with no cell lining } \\
\text { - RBCs perfused through system at } \\
\text { different concentrations } \\
\text { - } 0.4 \text { haematocrit }(30)\end{array}$ & $\begin{array}{l}\text { - Perfusion rate for RBCs stored for } \\
\text { different periods }(30)\end{array}$ & $\begin{array}{l}\text { - Perfusion rate for stored RBCs was } 19-30 \% \\
\text { lower than for fresh RBCs. Washing stored } \\
\text { RBCs in saline improved perfusion rate } \\
\text { by } 41 \%(30)\end{array}$ \\
\hline $\begin{array}{l}\text { Cellular synthetic } \\
\text { microvascular model }\end{array}$ & $\begin{array}{l}\text { - Soft lithography with vessel diameters } \\
\text { of } 50-200 \mu \mathrm{m} \text { in PDMS }(9) \text { or collagen }(31) \\
\text { - Channels lined with HUVECs }(9,31) \\
\text { - RBCs perfused at } 10-20 \%(\mathrm{v} / \mathrm{v}) \mathrm{RBCs} \\
\text { with shear stress up to } 17 \text { dyn/ } \mathrm{cm}^{2}(9,31) \\
\text { - Model can be stretched to simulate } \\
\text { respiratory forces }(9) \\
\text { - Perivascular cells can be added to collagen } \\
\text { matrix (31) }\end{array}$ & $\begin{array}{l}\text { - } \text { RBC adhesion, endothelial marker } \\
\text { expression, confocal microscopy ( } 31) \\
\text { - Endothelial damage }(9)\end{array}$ & $\begin{array}{l}\text { - Low hemodynamic shear stress due } \\
\text { to altered microcirculatory flow may } \\
\text { predispose HUVECs to necroptosis ( } 9 \text { ) } \\
\text { - Cyclic stretching of microvessels (similar to } \\
\text { breathing movements or mechanical } \\
\text { ventilation) may increase susceptibility of } \\
\text { HUVECs to transfused RBCs }(9)\end{array}$ \\
\hline
\end{tabular}

In vivo microvascular • Microvasculature assessed with

model (large animal) - Sublingual sidestream dark field imaging (46)

- Laser Doppler flowmeter (46)

- Tissue reflectance spectrophotometer (46)

- Animals used

- Domestic pig (46)
- Microcirculatory flow index, microvascular blood flow, capillary-venous hemoglobin oxygen saturation (46)
- Isotonic or hypertonic colloidal fluids adequately restored sublingual microcirculatory blood flow and flow quality (46)

- Gelatin + hydroxyethyl starch improved microvascular hemoglobin oxygen saturation (46)
In vivo microvascular model (small animal)
- Animals transfused with blood product and then assessed using intravital microscopy

- Animals used - Rat: cremaster flap (47), extensor digitorum longus muscle (48) - Mice cremaster flap (49)

- Hamster: dorsal skin flap (50)
- $\mathrm{RBC}$ velocity, vessel diameter, FCD, after stored PRBC transfusion (47)

- FCD, RBC velocity, vessel diameter, $\mathrm{O}_{2}$ distribution after PRBC transfusion (50)

- RBC adhesion in capillaries (48)

- $\mathrm{RBC}$ velocity, vessel diameter (49)
- Fresh PRBCs more effective at relieving microcirculatory hypoxia compared to stored PRBCs in rat cremaster flap model (47)

- IgG-mediated HTRs induced acute vaso-occlusive crisis in the mice cremaster model. CXCR2 blockage prevented HTR-induced vasoocclusive crisis (49)

BC: buffy coat; E. coli: Escherichia coli; FCD: functional capillary density; HTR: hemolytic transfusion reaction; HUVECs: human umbilical vin endothelial cells; LPS: lipopolysaccharide; PC: platelet concentrate; PDMS: polydimethylsiloxane; PMPs: platelet microparticles; PRBC: packed red blood cells; PRP: platelet-rich plasma; RBC: red blood cell; RMP: red cell microparticle; ROS: reactive oxygen species; TNF- $\alpha$ : tumour necrosis factor-alpha.

alignment. ${ }^{6}$ Lastly, shear stress modifies endothelial interactions with blood cells. For example, monocytes perfused over endothelium activated by tumor necrosis factor- $\alpha$ expressed more tissue factor and CD11b compared to monocytes co-incubated with activated endothelium under static conditions. ${ }^{9}$

It follows that intravascular transfusion events are affected by blood product-recipient blood interactions and blood mixture-endothelium interactions under flow conditions. Simulation of the post-transfusion intravascular mileau in vitro requires recipient whole blood, blood product, endothelium and perfusate flow. To date, the majority of models used to test transfusion effects have involved static models in which blood products are coincubated with specific cells such as neutrophils, ${ }^{1,17-21}$ macrophages, ${ }^{3}$ platelets ${ }^{22}$ or endothelial cells ${ }^{23}$ to examine interactions between two cell types. While blood co-incubation experiments enable the investigation of multiple cellular interactions, ${ }^{2,24}$ they do not recapitulate the frequency and type of cellular interactions that occur under endogenous flow conditions. In vivo models are the "gold standard" for capturing the sum of intravascular interac- tions that occur after transfusion of stored, packed RBCs. However, such models are limited by increased variability (requiring larger sample sizes), reduced capacity to isolate parameters and longer set-up times. Endothelialized in vitro flow models circumvent these limitations by using cell culture methods, packed RBCs and fresh whole blood, which are easier to acquire with fewer associated ethical considerations, shorter model development time and lower cost.

\section{What can we do with current manufacturing technology?}

The potential methods to investigate blood mixtureendothelial interactions and current research outputs from these models are summarized in Table 1. Flow models can be divided into planar, microvascular and macrovascular depending on the arrangement of endothelial and blood cells. For planar models, endothelial cells were cultured on coverslips and subjected to various shear stresses generated by laminar flow. ${ }^{25-27}$ These models were used to study blood-endothelial adhesion under macrovascular flow conditions with shear stresses of 0.3-10 dyn $/ \mathrm{cm}^{2} .25-27$ Notably, these models do not reca- 
pitulate macrovascular flow stream configurations (rectangular flow stream versus circular flow stream) and do not test physiological hematocrits $(<10 \%$ whole blood/packed RBC suspensions versus undiluted blood). ${ }^{25-}$
${ }^{27}$ The ex vivo artery model recapitulated physiological flow stream configurations by connecting human umbilical arteries in a perfusable circuit. ${ }^{28}$ However, the use of harvested arteries meant that the model was susceptible
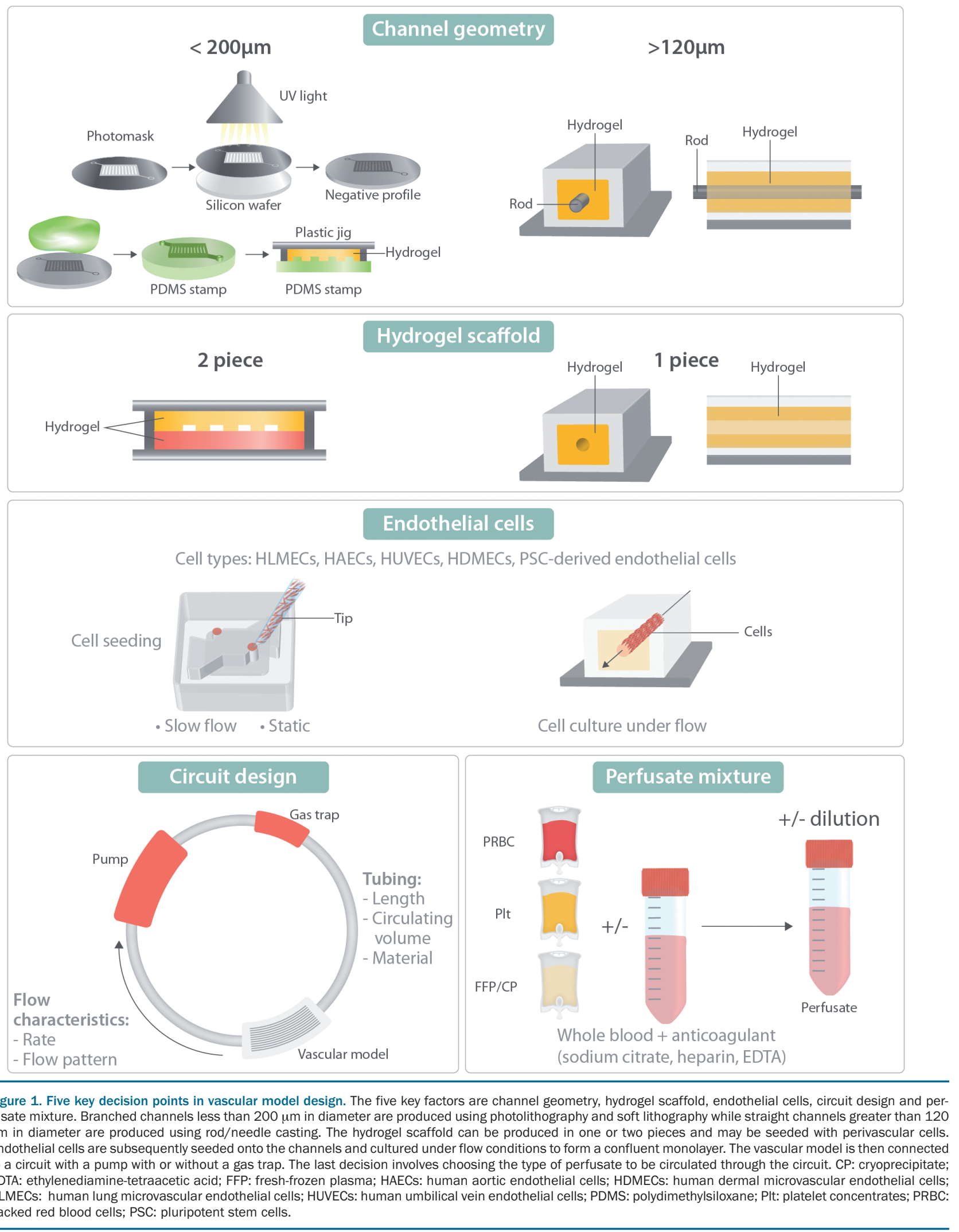
to inter-donor variation and ethical concerns. Synthetic arterial models, produced by seeding cells on a biodegradable tubular scaffold, can overcome these issues. ${ }^{29}$ To date, this model has not been perfused with blood to investigate blood-endothelial interactions.

Microvascular models involve the formation of $<200$ $\mu \mathrm{m}$ channels in polydimethylsiloxane, subendothelium or hydrogels. ${ }^{9,30,31}$ These channels are available in different branching geometries and can be lined by endothelial cells. ${ }^{31,32}$ Acellular models can accommodate smaller vessel diameters (5-70 $\mu \mathrm{m})$ and higher hematocrits (up to 0.4) without blockage. ${ }^{30}$ In contrast, endothelialized microvascular models are limited by vessel diameters of 50-200 $\mu \mathrm{m}$ with $10-20 \%$ (v/v) packed RBC perfusates. ${ }^{9,31}$ Vessels less than $50 \mu \mathrm{m}$ in diameter are difficult to produce in hydrogels, while vessels greater than $200 \mu \mathrm{m}$ present issues during endothelial seeding and culture which preclude the formation of a confluent monolayer. Manufacturing limitations associated with vessel diameter can be abrogated by using in vivo microvascular models such as muscle or dorsal skin flaps. However, these models lack the channel diameter and geometrical consistency resulting from in vitro-manufacturing techniques. The presence of endothelial cells in microvascular models enhances thrombosis, thereby hindering the hematocrits that can be tested. Physiological hematocrits are important for viscosity, shear stress and cell-to-cell interactions. For example, an increased hematocrit leads to more RBCendothelial interactions which enhance shear stress at low flow rates. Furthermore, an increased hematocrit has been associated with altered RBC arrangement in the flow stream and margination of blood components. ${ }^{33}$

\section{Designing vascular models}

Perfusable in vitro macrovascular and microvascular models are composed of an endothelialized channel in a hydrogel scaffold. The unit is subsequently connected to a circuit and perfused with the desired "test" fluid. There are five key decision points in vascular model design (Figure 1): (i) channel geometry, (ii) scaffold moulding, (iii) endothelial cell seeding, (iv) circuit construction, and (v) perfusate selection.

The choice of channel size and shape determines the scaffold manufacturing method. Straight channels with diameters down to $120 \mu \mathrm{m}$ can be injection-molded using rods, needles or wires stabilized on a mount. This method is cost-effective and technically easy to construct but has limited fidelity for endogenous vascular geometry. Branched channel geometries with diameters of 50$200 \mu \mathrm{m}$ can be formed using a mixture of photolithography and soft lithography methods. Photolithography is used to create a hard negative-profile wafer. Complex channel geometries can be etched onto a photomask which is used to develop the desired pattern on a photoresist-coated crystalline silicon wafer. ${ }^{34}$ Notably, photolithography requires microfabrication facilities (e.g. clean room, photo pattern generator, spin coater) and specialized consumables (e.g. crystalline silicon wafer, photoresist, developer). The negative-profile wafer becomes the mold for the soft positive-profile stamp used to shape the hydrogel scaffold by soft lithography. ${ }^{31}$ The soft positiveprofile stamp can be formed from polydimethylsiloxane in any standard laboratory using a vacuum degasser and oven. Polydimethylsiloxane can be printed directly in three dimensions: however, this method has inferior resolution compared to the photo/soft-lithography combination ( $760 \mu \mathrm{m}$ versus $50 \mu \mathrm{m}$, resolution limited by soft lithography). ${ }^{35}$

The hydrogel scaffold can be constructed from collagen, alginates, agarose, poly(ethylene glycol) dimethacrylate or methacrylated gelatin..$^{5}$ These materials are chosen for their transparency, absence of toxicity, fidelity for micropatterning and transport properties. Perivascular and/or tissue cells can be incorporated into the hydrogel to enhance biological approximation. The hydrogel is subsequently injection-molded onto a stamp and allowed to solidify at specified temperatures. Straight channel scaffolds can be formed as one piece. Scaffolds involving the use of a stamp are formed as two pieces (stamped pieced, flat slab), which are subsequently combined to form closed channels. The key obstacles at this stage are bubble formation during injection molding and channel damage during scaffold assembly.

The next decision involves the type of endothelial cells to use to seed the channels. Commercially available multi-donor human umbilical vein endothelial cells are the most commonly used cell type due to their availability, robustness and proliferation consistency. However, multi-donor cells can express multiple antigenic profiles, complicating donor-recipient cross-matching in transfusion simulations. Furthermore, human umbilical vein endothelial cells may behave differently from other primary endothelial cells. Vessel-specific primary endothelial cells such as human lung microvascular endothelial cells and human aortic endothelial cells may improve physiological relevance but these cells are often more difficult to culture and susceptible to variability. Endothelial derivatives from human pluripotent stem cells can also be used. ${ }^{36}$ The desired endothelial cells can be seeded on the channel surface under static or low flow conditions and grown to confluence under laminar flow. Notably, high seeding flow rates will prevent endothelial cells from attaching to the hydrogel scaffold. We recommend seeding endothelial cells under static conditions and allowing up to 3 hours for the cells to attach, prior to incubating the cells overnight under laminar flow conditions. Endothelial cell growth under laminar flow is important to cultivate the barrier function and monolayer present in endogenous blood vessels.

Variables in circuit construction include circulation volume, tubing material and diameter, gas trap and pump. The circulation volume can be adjusted to accommodate the desired outcome measures (e.g. flow cytometry, enzyme-linked immunosorbent assay, mass spectrometry, biochemical analysis). Tubing length and diameter can be varied to reach the desired circulation volume. Notably, the more tubing is used to maximize circulation volume, the greater the ratio between non-endothelialized and endothelized surface area in the circuit. Medical grade tubing made out of Tygon, fluoropolymers or polyvinylchloride are used as they are inert, biocompatible and gas permeable. In our set-up, the gas trap is connected just before the inlet port of the vascular model to minimize bubbles passing through the endothelialized 
portion of the circuit. The pump chosen needs to support the desired flow rate (macrovascular: $\leq 70 \mathrm{~mL} / \mathrm{min}$, microvascular: $\leq 50 \mu \mathrm{L} / \mathrm{min}$ ). Syringe pumps deliver consistent continuous flow rates. Peristaltic pumps can deliver continuous and pulsatile flow patterns. Due to the rotor mechanism of peristaltic pumps, the continuous flow setting may deliver a "dampened" pulsatile flow. Pumps which deliver inconsistent flow rates may lead to bubble formation in the circuit. The flow rate can be adjusted to simulate the desired environment (e.g. arteries, capillaries, veins) and shear stress. When calculating the shear rate, it is important to note that blood is a nonNewtonian fluid meaning that its viscosity and the shear stress that it exerts on the endothelium are dependent on the amount of pressure exerted on it. Lastly, the entire circuit can be placed in an incubator or the pump computer can be left outside the incubator (if there are ports available for the passage of cables).

Whole blood, blood product, whole blood-blood product mixtures and diluted blood can be perfused through the system to simulate blood flow. Undiluted blood best recapitulates the intravascular milieu by maintaining blood concentration and viscosity. However, undiluted anticoagulated whole blood still carries increased risks of thrombosis (particularly in microvascular channels) and is opaque - making it optically difficult to assess blood cell adhesion to endothelium. In these situations, blood can be diluted to maintain circulation and enable in situ microscopic analysis. Mixing whole blood with packed RBCs at a ratio of 9:1 simulates a one unit transfusion of packed RBCs. The whole blood should be diluted such that the hematocrit is below the transfusion threshold of $7 \mathrm{~g} / \mathrm{dL}$ to prevent post-transfusion hyperviscosity. While this dilution simulates the hemodilution observed after crystalloid resuscitation, it is unlikely to approximate the blood viscosity observed in patients with chronic anemia. Notably, all blood and blood products circulated through in vitro models are anticoagulated as this treatment is necessary for ex vivo blood storage prior to experimentation. Sodium citrate, ethylenediamine-tetraacetic acid (EDTA) and heparin anticoagulation are non-toxic and suitable for use. Sodium citrate and EDTA work by chelating calcium ions and their anticoagulant effect can be weakened by adding calcium. Sodium citrate is required for coagulation analyses. Furthermore, high levels of sodium citrate can alter the $\mathrm{pH}$ of blood, EDTA can activate endothelium and both chelators can interfere with microparticle formation. Heparin provides enduring anticoagulation, but it interferes with coagulation analyses and immune cell function. It follows that the requirement for anticoagulated blood represents a shortcoming of in vitro vascular models. This pitfall can be dealt with to some extent by using in vivo models to corroborate in vitro results.

Various outcome measures can be recorded from these in vitro vascular models. Real-time video microscopy can be used to visualize blood cell adhesion to the endothelium. The perfusant can be analyzed for soluble factors (e.g. cytokines, microparticles, biological response mediators) and cell surface markers. After blood circulation, the endothelial cells can be imaged in situ or removed from the scaffold with trypsin for further analysis. In our laboratory, a vascular model with a $3 \mathrm{~mm}$, full-length channel seeded with human umbilical vein endothelial cells is primarily used to simulate transfusion reactions by circulating mixtures of recipient whole blood and donor blood products (Online Supplementary Material). Whole blood circulation leads to increased formation of annexin V-positive microparticles and erythrocyte microparticles compared to statically held whole blood $\left(2.13 \times 10^{10}\right.$ versus $6.08 \times 10^{9}$ microparticles $/ \mathrm{mL}, \quad P<0.0001 \quad$ (Online Supplementary Material).

\section{Conclusion}

In vitro vascular models combine blood, endothelial and flow components into a single system. In this way, endothelialized flow models simulate the blood productrecipient blood and blood-endothelial interactions that occur under flow conditions after blood product transfusion. Five key factors of the experimental set-up (channel geometry, scaffold molding, endothelial cell seeding, circuit construction, and perfusate) can be manipulated depending on the desired organ system, transfusion scenario and outcome measures.

\section{References}

1 Jy W, Mao WW, Horstman L, Tao J, Ahn YS. Platelet microparticles bind, activate and aggregate neutrophils in vitro. Blood Cells Mol Dis. 1995;21(3):217-231.

2. Straat M, Boing AN, Tuip-De Boer A, Nieuwland R, Juffermans NP. Extracellular vesicles from red blood cell products induce a strong pro-inflammatory host response, dependent on both numbers and storage duration. Transfus Med Hemother. 2016;43(4):302-305.

3. Sadallah S, Eken C, Schifferli JA. Erythrocyte-derived ectosomes have immunosuppressive properties. J Leukoc Biol. 2008;84(5):1316-1325.

4. Karsten E, Herbert B. Red blood cells: the immune system's hidden regulator. Annual scientific meeting of Haematology Society of Australia and New Zealand, the Australian \& New Zealand Society of Blood Transfusion and the Thrombosis and Haemostasis Society of Australia and New Zealand. Sydney, Australia, 2017.

5. Rayner SG, Zheng Y. Engineered microvessels for the study of human disease. J Biomech Eng. 2016;138(11).

6. Li YS, Haga JH, Chien S. Molecular basis of the effects of shear stress on vascular endothelial cells. J Biomech. 2005;38(10):1949-1971.

7. Granger D, Senchenkova E. LeukocyteEndothelial Cell Adhesion. Inflammation and the Microcirculation. San Rafael (CA): Morgan \& Claypool Life Sciences, 2010.

8. Hack CE, Zeerleder S. The endothelium in sepsis: source of and a target for inflammation. Crit Care Med. 2001;29(7 Suppl):S2127

9. Seo J, Conegliano D, Farrell M, et al. A microengineered model of RBC transfusioninduced pulmonary vascular injury. Sci Rep. 2017;7(1):3413.

10. Hathcock JJ. Flow effects on coagulation and thrombosis. Arterioscler Thromb Vasc Biol. 2006;26(8):1729-1737.

11. Sakariassen KS, Orning L, Turitto VT. The impact of blood shear rate on arterial thrombus formation. Future Sci OA. 2015;1(4):FSO30.

12. Eckstein EC, Bilsker DL, Waters CM, Kippenhan JS, Tilles AW. Transport of platelets in flowing blood. Ann N Y Acad Sci. 1987;516:442-452.

13. Xu C, Wootton DM. Platelet near-wall excess in porcine whole blood in arterysized tubes under steady and pulsatile flow conditions. Biorheology. 2004;41(2):113125.

14. Dimmeler S, Haendeler J, Nehls M, Zeiher AM. Suppression of apoptosis by nitric 
oxide via inhibition of interleukin-1betaconverting enzyme (ICE)-like and cysteine protease protein (CPP)-32-like proteases. J Exp Med. 1997;185(4):601-607.

15. Miao H, Hu YL, Shiu YT, et al. Effects of flow patterns on the localization and expression of VE-cadherin at vascular endothelial cell junctions: in vivo and in vitro investigations. J Vasc Res. 2005;42(1):77-89.

16. Young EW, Watson MW, Srigunapalan S, Wheeler AR, Simmons CA. Technique for real-time measurements of endothelial permeability in a microfluidic membrane chip using laser-induced fluorescence detection. Anal Chem. 2010;82(3):808-816.

17. Biffl WL, Moore EE, Offner PJ, Ciesla DJ, Gonzalez RJ, Silliman CC. Plasma from aged stored red blood cells delays neutrophil apoptosis and primes for cytotoxicity: abrogation by poststorage washing but not prestorage leukoreduction. J Trauma. 2001:50(3):426-432.

18. Cardo LJ, Wilder D, Salata J. Neutrophil priming, caused by cell membranes and microvesicles in packed red blood cell units, is abrogated by leukocyte depletion at collection. Transfus Apher Sci. 2008;38(2):117-125.

19. Jank H, Salzer U. Vesicles generated during storage of red blood cells enhance the generation of radical oxygen species in activated neutrophils. ScientificWorldJournal. 2011;11:173-185.

20. Silliman CC, Moore EE, Kelher MR, Khan SY, Gellar L, Elzi DJ. Identification of lipids that accumulate during the routine storage of prestorage leukoreduced red blood cells and cause acute lung injury. Transfusion. 2011;51(12):2549-2554

21. Belizaire RM, Prakash PS, Richter JR, et al. Microparticles from stored red blood cells activate neutrophils and cause lung injury after hemorrhage and resuscitation. J Am Coll Surg. 2012;214(4):648-655; discussion 656-657.

22. Morrison A, McMillan L, Hornsey VS, Prowse CV. Stored red-blood-cells inhibit platelet function under physiologic flow. Vox Sang. 2010;99(4):362-368.

23. Bonomini M, Sirolli V, Gizzi F, Di Stante S, Grilli A, Felaco M. Enhanced adherence of human uremic erythrocytes to vascular endothelium: role of phosphatidylserine exposure. Kidney Int. 2002;62(4):1358-1363.

24. Karam O, Tucci M, Toledano BJ, et al. Length of storage and in vitro immunomodulation induced by prestorage leukoreduced red blood cells. Transfusion. 2009;49(11):2326-2334.

25. Macey MG, Wolf SI, Wheeler-Jones CP, Lawson C. Expression of blood coagulation factors on monocytes after exposure to TNF-treated endothelium in a novel whole blood model of arterial flow. I Immunol Methods. 2009;350(1-2):133-141.

26. Anniss AM, Sparrow RL. Storage duration and white blood cell content of red blood cell (RBC) products increases adhesion of stored RBCs to endothelium under flow conditions. Transfusion. 2006;46(9):15611567

27. Luk CS, Gray-Statchuk LA, Cepinkas G, Chin-Yee IH. WBC reduction reduces storage-associated RBC adhesion to human vascular endothelial cells under conditions of continuous flow in vitro. Transfusion. 2003;43(2):151-156

28. Holtom E, Usherwood JR, Macey MG, Lawson C. Microparticle formation after coculture of human whole blood and umbilical artery in a novel in vitro model of flow. Cytometry A. 2012;81(5):390-399.

29. Robert J, Weber B, Frese L, et al. A threedimensional engineered artery model for in vitro atherosclerosis research. PLoS One. 2013;8(11):e79821

30. Burns JM, Yang X, Forouzan O, Sosa JM, Shevkoplyas SS. Artificial microvascular network: a new tool for measuring rheologic properties of stored red blood cells. Transfusion. 2012;52(5):1010-1023

31. Morgan JP, Delnero PF, Zheng Y, et al. Formation of microvascular networks in vitro. Nat Protoc. 2013;8(9):1820-1836.

32. Chrobak KM, Potter DR, Tien J. Formation of perfused, functional microvascular tubes in vitro. Microvasc Re. 2006;71(3):185-196.

33. Muller K, Fedosov DA, Gompper G. Margination of micro- and nano-particles in blood flow and its effect on drug delivery. Sci Rep. 2014;4:4871

34. Martinez-Duarte R, Madou M. SU-8 Photolithography and its impact on microfluidics. 2010. In: Microfluidics and Nanofluidics Handbook: Fabrication, Implementation and Applications.pp239240.

35. Ozbolat V, Dey M, Ayan B, Povilianskas A, Demirel MC, Ozbolat IT. 3D Printing of PDMS improves its mechanical and cell adhesion properties. ACS Biomaterials Science \& Engineering. 2018;4(2):682-693.

36. Palpant NJ, Pabon L, Roberts M, et al. Inhibition of beta-catenin signaling respecifies anterior-like endothelium into beating human cardiomyocytes. Development. 2015;142(18):3198-3209.

37. Albanyan AM, Harrison P, Murphy MF Markers of platelet activation and apoptosis during storage of apheresis- and buffy coatderived platelet concentrates for 7 days. Transfusion. 2009;49(1):108-117.

38. Almizraq R, Tchir JD, Holovati JL, Acker JP. Storage of red blood cells affects membrane composition, microvesiculation, and in vitro quality. Transfusion. 2013;53(10):2258-2267.

39. Aucar JA, Isaak E, Anthony D. The effect of red blood cell age on coagulation. Am J Surg. 2009;198(6):900-904.

40. Bayer SB, Hampton MB, Winterbourn CC Accumulation of oxidized peroxiredoxin 2 in red blood cells and its prevention. Transfusion. 2015;55(8):1909-1918.

41. Karger R, Lukow C, Kretschmer V. Deformability of red blood cells and correlation with ATP content during storage as leukocyte-depleted whole blood. Transfus Med Hemother. 2012;39(4):277-282

42. Antonelou MH, Tzounakas VL, Velentzas $\mathrm{AD}$, Stamoulis KE, Kriebardis AG Papassideri IS. Effects of pre-storage leukoreduction on stored red blood cells signaling: a time-course evaluation from shape to proteome. J Proteomics. 2012;76 (Spec No.):220-238.

43. Fischer D, Bussow J, Meybohm P, et al Microparticles from stored red blood cells enhance procoagulant and proinflammatory activity. Transfusion. 2017;57(11):2701 2711.

44. Boomgaard MN, Gouwerok CW, Homburg $\mathrm{CH}$, de Groot G, IJsseldijk MJ, de Korte D The platelet adhesion capacity to subendothelial matrix and collagen in a flow model during storage of platelet concentrates for 7 days. Thromb Haemost. 1994;72(4):611-616

45. Anniss AM, Sparrow RL. Variable adhesion of different red blood cell products to activated vascular endothelium under flow conditions. Am J Hematol. 2007;82(6):439-445.

46. Maier S, Holz-Holzl C, Pajk W, et al Microcirculatory parameters after isotonic and hypertonic colloidal fluid resuscitation in acute hemorrhagic shock. J Trauma. 2009;66(2):337-345

47. Gonzalez AM, Yazici I, Kusza K, Siemionow M. Effects of fresh versus banked blood transfusions on microcirculatory hemodynamics and tissue oxygenation in the rat cremaster model. Surgery. 2007;141(5):630-639.

48. Chin-Yee IH, Gray-Statchuk L, Milkovich S, Ellis CG. Transfusion of stored red blood cells adhere in the rat microvasculature. Transfusion. 2009;49(11):2304-2310.

49. Jang JE, Hod EA, Spitalnik SL, Frenette PS. CXCL1 and its receptor, CXCR2, mediate murine sickle cell vaso-occlusion during hemolytic transfusion reactions. J Clin Invest. 2011;121(4):1397-1401.

50. Cabrales $\mathrm{P}$, Intaglietta $M$, Tsai AG Transfusion restores blood viscosity and reinstates microvascular conditions from hemorrhagic shock independent of oxygen carrying capacity. Resuscitation. 2007;75 (1):124-134 